\title{
VARIAÇÃO FONOLÓGICA E FONOLOGIA COMO GRAMÁTICA DE COMPREENSÃO ${ }^{1}$ PHONOLOGICAL VARIATION AND PHONOLOGY AS A COMPRE- HENSION GRAMMAR
}

\author{
Seung-Hwa Lee ${ }^{2}$
}

Resumo: $O$ sistema vocálico do português brasileiro apresenta contraste de 7 vogais na posição tônica. As vogais médias podem flutuar na posição pretônica $-\{\varepsilon \sim \mathrm{e} \sim \mathrm{i}, \mathrm{o} \sim \mathrm{0} \sim \mathrm{u}\}$ pelos processos fonológicos, como a harmonia vocálica de [Alto] ou [ATR] e a redução vocálica, e podem sofrer os processos fonológicos na posição tônica, cf. LEE (2012). Essas alternâncias nas vogais médias têm sido um dos temas mais discutidos na literatura. Este artigo discute a questão de variação/opcionalidade (fonológica) nos modelos anteriores e busca a elaboração de uma nova concepção de fonologia que inclui o conhecimento linguístico para a produção e a compreensão. Atentando para esse fato, este artigo propõe uma reinterpretação do componente fonológico na gramática, a partir do modelo proposto por Hale, Kissock and Reiss (2007).

Palavras-chave: Variação Fonológica, Fonologia, Vogais médias do PB. Compreensão

\begin{abstract}
Portuguese vowel system has 7 contrastive vowels in stressed syllable and medium vowels in unstressed syllable (pre-tonic position) can alternate among three different phonetic forms $-\{\varepsilon \sim \mathrm{e} \sim \mathrm{i}, \mathrm{o} \sim \mathrm{\rho} \sim \mathrm{u}\}-$ by [High]/[ATR] harmony from the stressed or adjacent vowels or by vowel reduction. This alternation in mid vowels is one of the most discussed topics in Brazilian Portuguese phonology and in Labovian sociolinguistic researches in Brazil. This paper discusses the phonological variation (optionality) in the previous theoretical grammar (phonology) models, like generative phonology and OT, which are production-based and unidirectional mapping model from input to output. And this paper proposes a new concept of phonological component to deal with the facts of variation both from the standpoint of production and comprehension.

Keywords: Phonological variation, Phonology, BP medium vowels, Comprehension

1 Agradeço a Prof. Marco Antônio de Oliveira, Profa. Leda Bisol e Profa. Maria Bernadete Abaurre pela discussão e comentários e a Profa. Eunice Nicolau pela leitura e correção do português. Todos os erros que persistem são da minha inteira responsabilidade.

2 Professor da Universidade Federal de Minas Gerais, pesquisador do CNPq.
\end{abstract}




\section{Introdução}

As vogais médias podem ter diferentes realizações fonéticas na posição pretônica no português brasileiro (doravante, $\mathrm{PB}$ ), dependendo da região ou do indivíduo e essas vogais flutuam em relação ao traço [ATR] e ao traço [Alto], que são resultados de Harmonia Vocálica, Redução Vocálica ou de mapeamento fiel $-\{\varepsilon \sim \mathrm{e} \sim \mathrm{i}, \mathrm{o} \sim 0 \sim \mathrm{u}\}$. Lee e Oliveira (2006) mostram que essas alternâncias vocálicas do $\mathrm{PB}$ trazem problemas tanto para uma teoria de cunho formal (CHOMSKY e HALLE, 1968; e PRINCE e SMOLENSKY, 1993) quanto para uma teoria de cunho variacionista (WEINREICH, LABOV e HERZOG, 1968 e LABOV, 1969) - uma vez que existe assimetria entre compreensão e produção; ou seja, o falante nativo compreende as formas fonéticas da comunidade, mas produz somente uma delas.

A variação fonológica não recebeu atenção nas teorias formais da fonologia. Os estudos da fonologia, desde o Estruturalismo, se concentraram na derivação da representação superficial a partir de uma representação subjacente - o mapeamento do input para output. Além disso, nos modelos das teorias formais (Fonologia Gerativa, CHOMSKY e HALLE, 1968; Teoria da Otimalidade Clássica (doravante, OT), PRINCE e SMOLENSKY, 1993), somente um output (forma fonética correta) é escolhido a partir de um input dado - modelo unidirecional - e, diferentemente, nos casos da variação fonológica, duas ou mais formas são escolhidas para um input dado. A variação fonológica é tratada como a opcionalidade de aplicação das regras fonológicas.

Este artigo rediscute as questões levantadas nos trabalhos anteriores sobre a flutuação das vogais médias do PB e propõe uma nova concepção de fonologia que se baseia tanto na produção quanto na compreensão.

\section{Alguns Fatos sobre as vogais do PB}

O sistema vocálico do português brasileiro apresenta contraste de sete vogais na posição tônica (cf. CÂMARA JR, 1970), como os exemplos (1) demonstram:

(1)

$S[\varepsilon]$ de vs. $s[e]$ de vs. $c[i]$ de

S[ว]co vs. s[o]co vs. s[u]co (extraído de LEE, 2012)

Através deste contraste/distinção na posição tônica, os estudos realizados sobre a fonologia do português assumem que existem sete vogais na representação subjacente (no inventário fonêmico). Apesar do contraste 
fonêmico apresentado acima, no entanto, as vogais na posição tônica podem sofrer alternâncias no português e mostram a relação alofônica entre as vogais médias. Nos não verbos ${ }^{3}$, por exemplo, a metafonia muda as vogais médias baixas para as vogais médias altas (cf. MIRANDA, 2007), como os exemplos abaixo mostram.

(2)

gost[o]so vs. gost[つ]sas vs. gost[○]sos

$\mathrm{P}[\mathrm{o}] \mathrm{rco}$ vs. $\mathrm{p}[\mathrm{o}] \mathrm{rca}$ vs. $\mathrm{p}[\mathrm{o}] \mathrm{rcos}$

E a conjugação verbal do português apresenta a relação alofônica entre as vogais médias e as vogais altas (cf. LEE, 2012).

(3)

$$
\mathrm{s}[\varepsilon] \mathrm{rvi}+\mathrm{o}=>\mathrm{s}[1] \mathrm{rvvo} \quad \mathrm{s}[\varepsilon] \mathrm{rvi}+\mathrm{a}=>\mathrm{s}[\mathbf{i}] \mathrm{rva}, \mathrm{s}[\mathrm{e}] \mathrm{rvi}+\mathrm{mos}
$$

Os processos fonológicos que ocorrem no português são categóricos e ocorrem durante a fonologia lexical (KIPARSKY, 1982) ${ }^{4}$ e sempre as vogais médias baixas são alvo para os processos fonológicos que envolvem os traços de [Alto] e [ATR].

Nas posições átonas, o sistema vocálico do $\mathrm{PB}$ apresenta 3 vogais na posição final de palavra - $\{e, I, U\}$ e as flutuações das vogais médias na posição pretônica $-\{\varepsilon \sim \mathrm{e} \sim \mathrm{i}, \mathrm{o} \sim \mathrm{0} \sim \mathrm{u}\}$. As realizações fonéticas do português na posição átona podem ser resumidas como em (4).

(4)

$$
\begin{aligned}
& / \mathrm{i} /=[\mathrm{i}],[\mathrm{I}] \\
& / \mathrm{e} / \rightarrow[\mathrm{e}],[\mathrm{\varepsilon}],[\mathrm{i}] \text { ou }[\mathrm{I}] \\
& / \varepsilon /->[\mathrm{e}],[\varepsilon],[\mathrm{i}] \text { ou }[\mathrm{I}] \\
& / \mathrm{u} /->[\mathrm{u}],[\mathrm{U}] \\
& / \mathrm{o} / \rightarrow>[\mathrm{o}],[\mathrm{U}],[\mathrm{u}] \text { ou }[\mathrm{o}] \\
& / \mathrm{o} /->[\mathrm{o}],[\mathrm{o}],[\mathrm{u}] \text { ou }[\mathrm{U}] \\
& / \mathrm{a} /->[\mathrm{a}],[\mathrm{e}]
\end{aligned}
$$

O mapeamento das vogais do $\mathrm{PB}$, acima, mostra que a vogal baixa e as vogais altas sempre preservam seus traços na forma fonética (mapeamento fiel), enquanto as vogais médias podem alternar em três formas fonéticas distintas. Esta flutuação na posição átona é variável e alofônica (cf. LEE e OLIVEIRA, 2006).

3 As vogais médias podem sofrer o abaixamento datílico e o abaixamento espondaico na posição tônica (cf. WETZELS, 1992).

4 Coetzee e Pater (2012) utilizam o termo early phonology.

Organon, Porto Alegre, v. 28, n. 54, p. 101-113, jan./jun. 2013. 
(5)

Relação Alofônica na sílaba pretônica

$\mathrm{R}[\mathrm{e}]$ cibo $\sim \mathrm{r}[\varepsilon]$ cibo $\sim \mathrm{r}[\mathrm{i}]$ cibo

$\mathrm{m}[\mathrm{o}] \mathrm{d} \varepsilon$ rno $\sim \mathrm{m}[\mathrm{o}] \mathrm{d} \varepsilon \mathrm{rno} \sim \mathrm{m}[\mathrm{u}] \mathrm{d} \varepsilon$ rno (LEE e OLIVEIRA, 2006)

As alternâncias das vogais médias também envolvem os traços de [Alto] e [ATR] como ocorrem nos processos fonológicos categóricos - harmonia vocálica de [Alto] e harmonia vocálica de [ATR].

(6)
a. Harmonia Vocálica de [ATR]
[perere'ke], b[e]leza
b. Harmonia Vocálica de [+Alto]
$\mathrm{m}[\mathrm{i}]$ nino

Também pode se aplicar a redução vocálica (6b) ou abaixamento vocálico (6a).

(7)
a. $\mathrm{p}[\supset]$ mada, $\mathrm{p}[\varepsilon]$ sada
b. $\mathrm{m}[\mathrm{u}]$ derno, c[u]légio

Os processos fonológicos variáveis modificam os traços de [ATR] e/ ou [Alto] das vogais médias da representação subjacente e estes processos aplicam-se no domínio de palavra.

Em resumo, os fatos mencionados acima mostram que a vogal baixa é neutra em termos de processos fonológicos; as vogais altas sempre engatilham e as vogais médias - altas e baixas - sofrem e engatilham esses processos. Lee (2008) mostra que estes fatos podem ser explicados através da hierarquia contrastiva dos traços no inventário das vogais do PB - Baixo $>$ Recuado $>$ Alto $>$ ATR. Essa teoria, no entanto, apenas determina o inventário, mas não explica a variação/flutuação das vogais médias no PB.

\section{Variação fonológica e Modelo da Gramática}

A flutuação das vogais médias na posição átona tornou-se um dos tópicos mais discutidos nos estudos da fonologia e nos estudos da sociolinguística variacionista nos últimos anos no Brasil.

Lee e Oliveira (2006) mostram que a flutuação nas vogais médias em posição pretônica envolve questões ainda mais complexas, como mistura 
de atuação de processos fonológicos, casos de realização categórica, falta de variação e mudança categórica de classe e compreensão comum. Os exemplos são repetidos em (8)-(10) para subsidiarem a discussão adiante: (8)

Mistura dos processos fonológicos e pronúncia categórica

a. Falante A: [bulitfí́], [dzidáu], [modéhnu]

b. Falante B: [boletfí], [dedáu], [modéhnu]

c. Falante C: [bulitfí'], [dedáu], [modéhnu]

d. Falante D: [bulitfí'], [dzidáu], [mudéhinu]

Os exemplos de (8) mostram que os falantes nativos escolhem aleatoriamente um dos processos fonológicos que satisfazem o contexto, e sua pronúncia é categórica nos falares em Belo Horizonte. Os exemplos de (9), abaixo, mostram que a variação fonológica (opcionalidade) não ocorre - somente uma forma fonética é possível, embora o contexto fonológico favoreça a opcionalidade:

(9)

Falta de variação (BH-MG)

a. Redução Vocálica

[tumát $\left.\int \mathrm{I}\right]{ }^{*} \mathrm{t}[\mathrm{o}]$ mate vs. [tomáde] ${ }^{*}[$ tumáde]

$\mathrm{t}[\mathrm{i}]$ soura $* \mathrm{t}[\mathrm{e}]$ souroa vs; $\mathrm{t}[\mathrm{e}]$ souro $* \mathrm{t}[\mathrm{i}]$ souro

b. Harmonia Vocálica de [+alto]

$\mathrm{c}[\mathrm{o}] \mathrm{riza}{ }^{*} \mathrm{c}[\mathrm{u}]$ riza vs. ${ }^{*}$ corisco curisco

$\mathrm{v}[\mathrm{i}]$ stido vs $* \mathrm{v}[\mathrm{e}]$ stido, v[e]stígio v[i]stígio

As palavras listadas em (10) não apresentam a variação fonológica das vogais médias nos falares mineiros, nos quais, a vogal alta e a vogal média na posição pretônica constituem categorias diferentes. Mesmo assim, os falantes fora de Minas Gerais não apresentam dificuldades para entender esta mudança, embora eles não pronunciem estas palavras como os mineiros:

Mudança categórica de classe/compreensão comum $\mathrm{p}[\mathrm{u}] \mathrm{rção} \mathrm{(muitas)} \quad \mathrm{p}[\mathrm{o}] \mathrm{rção} \mathrm{(uma} \mathrm{certa} \mathrm{quantidade)}$

$\mathrm{f}[\mathrm{u}]$ lhinha (calendário) $\mathrm{f}[\mathrm{o}]$ lhinha (folha pequena)

f[u]gão (eletrodoméstico) f[o]gão (fogo grande)

$\operatorname{pr}[\mathrm{i}]$ ciso (adjetivo) $\quad \operatorname{pr}[\mathrm{e}]$ ciso (verbo)

Lee e Oliveira (2006) observam que os exemplos de (8-10) trazem problemas tanto para as teorias de cunho social (sociolinguística laboviana) quanto para as teorias formais de fonologia, como fonologia gerati- 
va (CHOMSKY e HALLE, 1968) e a OT (PRINCE e SMOLENSKY, 1993; MCCARTHY e PRINCE, 1995).

A variação fonológica é tratada pelas regras opcionais e essas regras aplicam-se no componente pós-lexical (late phonology) na tradição da fonologia gerativa - ou seja, a variação fonológica faz parte do desempenho linguístico (Performance). O modelo da fonologia gerativa utiliza a regra de escrita para explicar/descrever a gramática e assume dois níveis da representação.

$$
\mathrm{A}->\mathrm{B} / \mathrm{C} \_\mathrm{D}
$$

/Representação Subjacente/ -> [Representação Fonética]

$\mathrm{Na}$ descrição da gramática (fonologia), este modelo baseia-se na produção, de modo que é unidirecional, tendo como foco: o elemento (A) que sofre a mudança, e o contexto $\left(C \_D\right)$ onde a mudança ocorre. A variação fonológica é explicada através da opcionalidade de aplicação de regras. No entanto, os exemplos de (9) mostram que as regras variáveis não se aplicam, embora o contexto as satisfaça - as regras apresentam exceções na sua aplicação, como prevê a fonologia lexical (KIPARKSY, 1982). Além disso, os processos fonológicos na posição átona ocorrem no domínio de palavra que pertence no componente lexical (early phonology) - as alternâncias não se aplicam através das palavras no português.

A sociolinguística laboviana utiliza as regras variáveis e probabilísticas que fazem parte do desempenho linguístico. Lee e Oliveira (2006) mostram, contudo, que a vertente social enfrenta os mesmos problemas que a fonologia gerativa na explicação da variação fonológica das vogais do PB.

Por outro lado, a OT concentra seu esforço para avaliar B na regra (11) - ou seja, o foco da OT para a descrição da gramática é CBD (mudança de estrutura), enquanto o da fonologia gerativa é CAD (descrição de estrutura). Este fato mostra um caminho natural para o aumento do interesse pela variação fonológica na abordagem da OT nos últimos anos. No entanto, Oliveira e Lee (2008) mostram que, a OT, embora seja considerada uma das teorias formais mais apropriadas para explicar a variação linguística (ou opcionalidade), ela não consegue explicar as alternâncias das vogais médias do PB, uma vez que assume o modelo unidirecional de Input para output que se baseia no modelo da produção, herdado do modelo da fonologia gerativa. A OT explica a opcionalidade em termos de função da EVAL (Ranqueamento Livre, Empate entre as restrições, Ranqueamento Parcial (cofonologia) e Ranqueamento Ordenado, etc.). Nestes modelos 
de EVAL (no caso da opcionalidade), a gramática da OT tenta obter todas formas possíveis (output) a partir de um input dado. Estes modelos também não conseguem explicar os exemplos de (8-10) acima: i) por que somente uma entre três formas possíveis?; ii) por que a escolha do processo fonológico é aleatória?; iii) por que há exceções?; iv) por que os falantes se entendem apesar de gramáticas diferentes?

Outra tentativa da OT, que incorpora o conceito de percepção e a probabilidade na gramática da OT, como Boersma (1997), Coetzee e Pater (2012), também não dá conta dos casos da mistura de processos fonológicos - a escolha aleatória de um processo fonológico entre três processos e sua pronúncia categórica após a escolha ${ }^{5}$. Nestes trabalhos a variação fonológica é tratada como opcionalidade - duas ou mais formas fonéticas possíveis no output. Os dados discutidos sobre a flutuação das vogais médias na posição pretônica do $\mathrm{PB}$ mostram que a variação fonológica não depende da opcionalidade. A opcionalidade (outputs múltiplos) ocorre na comunidade linguística e não ocorre sempre num mesmo indivíduo. Além disso, os exemplos de (10) não apresentam a variação fonológica, embora as vogais médias satisfaçam os contextos para se aplicar os processos fonológicos. Isso mostra que a variação fonológica não é contínua e probabilística.

Quando se trata da variação interdialetal, o problema é mais complexo. Lee (2012) mostra que a vogal média baixa é resultado de processos fonológicos diferentes - é oriunda de harmonia vocálica de traço [ATR] em Minas (Belo Horizonte) e pode ser de harmonia vocálica ou de abaixamento vocálico em Pernambuco (Recife). Isso leva a perguntar: o falante nativo mineiro precisaria ter regra de abaixamento vocálico na posição pretônica para compreender o falante nativo pernambucano? E mais, não existem estes processos fonológicos (harmonia vocálica de [ATR] e abaixamento vocálico) nos falares paulistas e sulistas, mas um falante nativo destas regiões não apresenta nenhum problema para se comunicar com os falantes com vogal média baixa na posição pretônica. De acordo com a fonologia gerativa e a OT, a vogal média baixa para estes falantes é agramatical, uma vez que eles nunca produzem esta vogal. Como este conhecimento pode ser incluído na gramática? Os falantes do sul e de São Paulo possuem somente cinco vogais na posição pretônica, enquanto os de Minas e de Pernambuco apresentam sete vogais nesta posição. Mesmo assim, os falantes se entendem bem uns com outros. Lee e Oliveira (2006) mostram que os modelos baseados na produção não são suficientes e sugerem a inclusão da percepção ${ }^{6}$ na gramática.

5 Lee e Oliveira (2006) mostram que a variação é mínima, quando se trata da fala de um indivíduo. É provável que seja um caso de microvariação (cf. HALE, KISSOCK and REISS, 2007) durante a implementação fonética (no componente de transdutor articulatório), quando ela ocorre.

6 De acordo com o modelo proposto por Hale, Kissock and Reiss (2007), a percepção não faz 


\section{Fonologia como a gramática de compreensão}

Os trabalhos anteriores nas teorias da fonologia, como no modelo gerativo clássico, nos modelos não lineares e na OT, se baseiam na produção, concebendo a gramática como unidirecional, partindo de uma representação subjacente para uma representação de superfície. No entanto, não há espaço para a compreensão, que pode produzir julgamentos sobre a gramaticalidade das formas possíveis, embora um indivíduo, numa determinada língua, nunca produza algumas das formas entre as formas possíveis.

O modelo da fonologia proposto por Hale, Kissock and Reiss (2007) abaixo separa o componente da implementação fonética em dois componentes paralelos - audição/percepção e articulação. De acordo com este modelo, a fonologia envolve somente o mapeamento de traços para traços e os componentes são estritamente modulares - nenhum componente pode enxergar outro componente, mas o componente fonológico alimenta o componente de transdutor ${ }^{7}$ articulatório, mas o componente de transdutor articulatório não alimenta o componente de percepção.

Fonologia: Representação Subjacente (UR) $\leftrightarrow$ Representação Superficial (SR)

Transdutor perceptual: SR $\leftarrow$ Valores Acústicos (Acoustic Score)

Transdutor articulatório: SR $\rightarrow$ Valores Gestuais (Gestural Score)

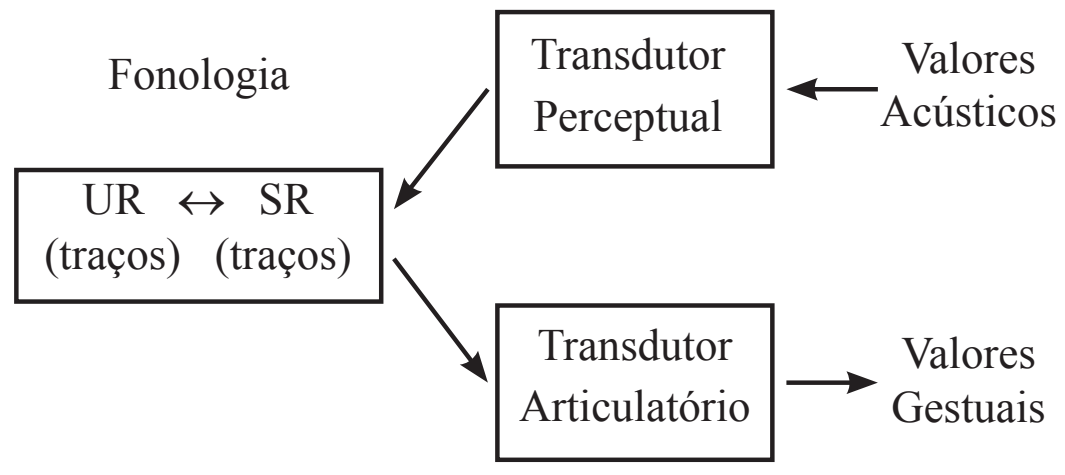

No modelo acima, portanto, a percepção e a articulação fazem parte da implementação fonética e não fazem parte da fonologia; elas evolvem dois processamentos unidirecionais que funcionam diferentemente - a articulação converte os traços em valores gestuais (e acústicos) e a audição/

parte da fonologia. Assume-se, então, neste artigo que a percepção (LEE e OLIVEIRA, 2006) seja entendida como a compreensão no componente fonológico.

7 Este modelo assume que os dois transdutores, ou conversores, - articulatório e perceptual - são inatos, invariáveis e idênticos para todos os seres humanos. 
percepção converte os valores acústicos em traços. Existe falta de isomorfia entre a articulação e a audição - dois sons de categorias diferentes podem apresentar os valores acústicos iguais ou semelhantes, mas o falante percebe/identifica as diferentes categorias de som respectivamente, apesar dos mesmos valores acústicos. As análises acústicas das vogais do NURC (MORAES et al, 1996), por exemplo, mostram que a vogal [u] em Recife apresenta valores acústicos bem semelhantes ao da vogal [o] em Salvador - os valores acústicos das duas vogais se sobrepõem, mas os falantes distinguem as duas vogais sem dificuldades.

Este modelo implica em que o componente fonológico seja responsável não somente pela produção, mas também pela percepção para julgar a gramaticalidade das formas. Os dados discutidos em (8)-(10) mostram assimetria entre a produção e a compreensão sobre a flutuação das vogais médias na posição pretônica. Além disso, os processos fonológicos discutidos na seção 1 mostram que a flutuação das vogais médias é caso de variação fonológica, no qual os traços de representação subjacente mudam para outros traços na representação superficial (fonética). Em outras palavras, a variação fonológica deve ser resolvida em termos da fonologia.

Este artigo, portanto, propõe que a teoria fonológica deve ser mais abrangente do que se propõe nos modelos gerativos (inclusive, na OT), nos quais a fonologia se baseia na produção, é unidirecional e não disponibiliza o espaço para a compreensão. Além disso, a fonologia deve explicar o conhecimento linguístico do falante nativo, que envolve as formas possíveis numa determinada língua, embora algumas destas formas não sejam produzidas por todos os falantes nativos dessa mesma língua - caso da variação fonológica.

O quadro abaixo mostra o caso de variação fonológica do $\mathrm{PB}$, onde a fonologia prevê as formas possíveis das vogais médias subjacentes: $\{\varepsilon \sim \mathrm{e} \sim$

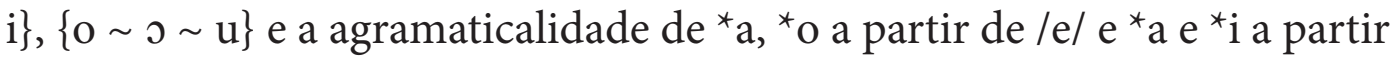
de $/ \mathrm{o} /$.

Fonologia

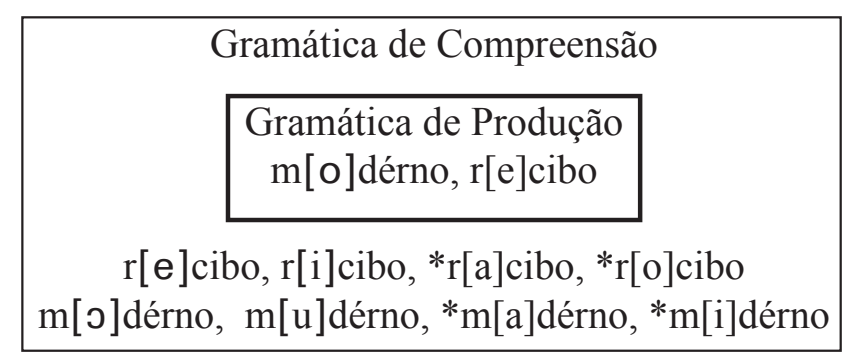

Organon, Porto Alegre, v. 28, n. 54, p. 101-113, jan./jun. 2013. 
Assume-se, aqui que a gramática da compreensão se baseia nos princípios não violáveis e gerais em cada língua. Oliveira (2011) propõe, para o sistema vocálico do $\mathrm{PB}$, o princípio, abaixo, que se incluiria na gramática de compreensão.

(15) Em posição átona, discrimine primeiramente [ $\alpha$ BAIXO] e [ $\alpha$ RECUADO $]^{8}$.

Este princípio pode ser reescrito como Ident [BAixo], Ident [RecUADO] em termos da OT. O tableau ${ }^{9}$ abaixo mostra que as restrições nãodominadas, IdENT [BAIXo], IDENT [RECUADo], garantem três formas possíveis no $\mathrm{PB}$.

(16)

\begin{tabular}{|c|c|c|c|}
\hline /moderno/ & $\begin{array}{l}\text { IDENT [BAIXo], IDENT } \\
\text { [RECUADO] }\end{array}$ & $\begin{array}{c}\text { Agree[ATR] }]_{1}^{*} \text { Mid IIDENT(Altura) } \\
1\end{array}$ & ildENT [ATR] \\
\hline$\$$ a. moderno & & $1^{*}$ & I \\
\hline b. moderno & & $1^{*}$ & $i^{*}$ \\
\hline$\sigma^{\circ} \mathrm{c}$ muderno & & $11^{*}$ & 1 \\
\hline
\end{tabular}

Por outro lado, a gramática de produção seleciona uma das três formas possíveis. Lee e Oliveira (2006) mostram que esta realização se baseia no item lexical/indivíduo. Como foi observado nos exemplos de (8), o falante nativo escolhe um dos processos fonológicos disponíveis na sua produção e os tableaux abaixo mostram como a gramática da produção funciona em termos da OT:

/moderno/ -> [moderno]

\begin{tabular}{|c|c|c|c|c|}
\hline /moderno/ & $\begin{array}{l}\text { IDENT } \quad \text { [BAIXo] } \\
\text { IDENT }\end{array}$ & AgreE[ATR] & $\begin{array}{c}{ }^{*} \text { Mid IIDENT(ALTURA) } \\
\vdots \\
1\end{array}$ & iIDENT [ATR] \\
\hline a. moderno & & ${ }^{* !}$ & 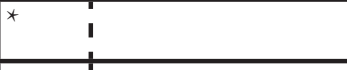 & $i$ \\
\hline b. moderno & & & i & $i^{*}$ \\
\hline c. muderno & & *! & $1^{*}$ & $!$ \\
\hline
\end{tabular}

8 Este artigo segue a proposta de Lee (2008 e 2009), na qual os traços das vogais são hierarquizados no inventário $-[$ Baixo $]>[$ Recuado $]>[$ Alto $]>[$ ATR $]$.

\begin{tabular}{|l|l|l|l|l|l|l|l|}
\hline & i & e & $\varepsilon$ & a & o & o & u \\
\hline Baixo & - & - & - & + & - & - & - \\
\hline Recuado & - & - & - & & + & + & + \\
\hline Alto & + & - & - & & - & - & + \\
\hline ATR & & + & - & & - & + & \\
\hline
\end{tabular}

9 Somente as violações referente às vogais na posições são computadas nos tableaux (16) - (18). 
/moderno/ -> [moderno]

\begin{tabular}{|c|c|c|c|c|}
\hline /moderno/ & $\begin{array}{l}\text { IDENT [BAIXO], IDENT } \\
\text { [RECUADO] }\end{array}$ & IDENT(ALTURA):IDENT [ATR] & AGREE[ATR] & $i^{\star}$ MID \\
\hline a. moderno & & ! & * & $i^{*}$ \\
\hline b. məderno & & i*! & & $i^{\star}$ \\
\hline c. muderno & & ${ }^{\star !} !$ & * & 1 \\
\hline
\end{tabular}

/moderno/ -> [muderno]

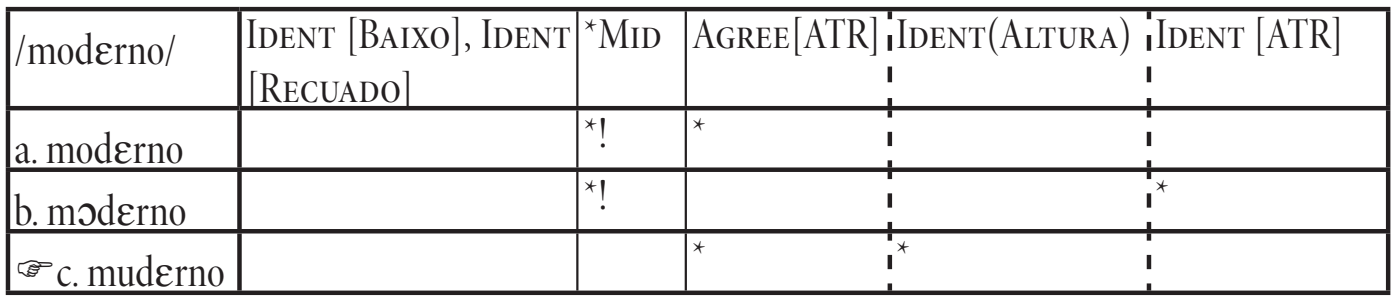

A expansão da concepção de fonologia traz algumas consequências interessantes em termos de teoria. A variação fonológica não se sujeita ao componente pós-lexical (late phonology); mas sim ao componente lexical (early phonology). Isso significa que a variação (fonológica) faz parte da Gramática-I não da Gramática-E (cf. Chomsky, 1986) e não é a questão da produção na forma fonética, mas da compreensão (cf. Oliveira, 2009). Portanto, a fonologia é o componente responsável pelas formas possíveis que não correspondem necessariamente à produção - a gramática da produção é o subconjunto da gramática da compreensão.

\section{Considerações finais}

Este artigo discutiu a variação fonológica e os modelos da fonologia. A variação fonológica foi tratada como o resultado do desempenho linguístico (late phonology) na maioria das vezes e como o resultado da questão de opcionalidade na produção. Os dados da flutuação das vogais médias na posição pretônica do $\mathrm{PB}$ mostram que a variação fonológica deve ser tratada no componente lexical (early phonology) e os modelos linguísticos (de cunho formal e de cunho social) não conseguem explicar estes dados satisfatoriamente, na medida em que assumem uma gramática unidirecional. Diante desse fato, propõe um conceito mais abrangente para o papel do componente fonológico - a fonologia deve tratar todas as formas possíveis numa determinada língua, embora o falante nativo desta língua não produza algumas dessas formas possíveis, como nos casos de mistura dos processos e escolha aleatória do processo fonológico. Assim, a variação 
fonológica faz parte da gramática da compreensão; não da gramática da produção. E, nessa proposta, a gramática da produção é subconjunto da gramática da compreensão - consequentemente, a variação fonológica faz parte da Língua-Internalizada.

\section{BIBLIOGRAFIA}

BOERSMA, Paul. How we learn variation, optionality, and probability. Proceedings of the Institute of Phonetic Sciences of the University of Amsterdam. 21, 1997. p: 43-58.

CÂMARA JR., Mattoso. Estrutura da Língua Portuguesa. Vozes. Petrópolis, Rio de Janeiro. 1970.

CHOMSKY, N. Knowledge of Language: Its Nature, Origin, and Use. Praeger, Westport, Conn., 1986.

CHOMSKY, Noam; HALLE, Morris. The Sound Pattern of English. New York: Harper e Row, 1968.

COETZEE, Andries W.; PATER, Joe. The place of variation in phonological theory. In: GOLDSMITH, John; RIGGLE, Jason; YU, Alan (eds.) The Handbook of Phonological Theory. 2nd Edition. Oxford: Blackwell, 2012. p. 401-434.

HALE, Mark; KISSOCK, Madelyn; REISS, Charles. Microvariation, variation, and the features of universal grammar. Lingua, 117, 2007. p.645-665. LABOV, William. Contraction, deletion, and inherent variability of the English copula. Language, 1969, 45, 715-762. Reimpresso, com modificações, em LABOV, William. Language in the inner city. Philadelphia: University of Pennsylvania Press, 65-129, 1972.

KIPARSKY, Paul. Lexical phonology and morphology. In: YANG, In-Seok (ed.) Linguistics in the Morning Calm. Seoul: Hanshin, 1982. p. 1-91.

LEE, Seung-Hwa . Contraste das Vogais no PB. Portuguese-Brazilian Studies, v. 5, Seoul. Korea. p. 201-221, 2008.

LEE, Seung-Hwa. Contraste das Vogais no PB e OT. Estudos Linguísticos, v. 30, p. 35-44, 2010.

LEE, Seung-Hwa. Variação Fonológica e Contraste no sistema vocálico do PB. In: LEE, Seung-Hwa (Org.) Vogais Além de Belo Horizonte. 2012. Editora FALE/UFMG.

LEE, S-H. e OLIVEIRA, Marco Antônio de. Phonological Theory and Language Variation in BP Mid Vowel Alternation. In: Proceeding of SICOL 2006. Seoul: The Linguistic Society of Korea, 2006. v. 1. p. 298-306. 
MCCARTHY, J.; PRINCE, A. Faithfulness and reduplicative identity in Prosodic Morphology. In: BECKMAN, DICKEY E URBANCZYK (eds.). Papers in Optimality Theory. pp. 249-384. Amherst, MA: GLSA. 1995.

MIRANDA, Ana Ruth Moresco. A metafonia nominal do Português do Brasil à luz da Teoria da Otimidade. Texto apresentado no III Seminário Internacional de Fonologia. Porto Alegre. 2007.

MORAES, João; CALLOU, Dinah; LEITE, Yonne. O sistema vocálico do português do Brasil: caracterização acústica. In: KATO, Mary. A. (org.). Gramática do português falado. São Paulo: Editora da UNICAMP/FAPESP, 1996. v. V: Convergências. p. 33-53.

OLIVEIRA, Marco Antonio de. Variação fonológica: o indivíduo e a comunidade de fala. In: CORTINA, Arnaldo; NASSER, Sílvia Maria Gomes da Conceição. (org.). Sujeito e linguagem. 17ed. São Paulo: Cultura Acadêmica, 2009, v. 1, p. 97-116.

OLIVEIRA, Marco Antonio de. Variação fonológica: o indivíduo e o léxico como atratores na perspectiva dos sistemas complexos. In: SILVA, Augusto Soares da; TORRES, Amadeu; GONÇALVES, Miguel. (Org.). Línguas Pluricêntricas - Variação linguística e dimensões sociocognitivas. $1^{\mathrm{a} e d .}$ Braga: ALETHEA - Associação Científica e Cultural, 2011, v. 01, p. 669-680. OLIVEIRA, Marco Antonio de e LEE, Seung Hwa. Variação Lingüística, Teoria Fonológica e Difusão Lexical. In: XV Congresso Internacional da Associação de Lingüística e Filologia da América Latina, Montevideo. XV ALFAL, 2008.

PRINCE, A.; SMOLENSKY, P. Optimality Theory: Constraints Interaction in Generative Grammar. Report No. RuCCS-TR-54, New Brunswick, NJ: Rutgers University Center for Cognitive Science, 1993.

WEINREICH, U.; LABOV, W.; HERZOG, M.. Empirical foundations for a theory of language change. In: LEHMANN, W. P.; MALKIEL, Y. (eds.) Directions for historical linguistics. Austin: University of Texas Press, 1968. p. 85-195.

WETZELS, W. Leo. Mid Vowel Neutralization. in Brazilian Portuguese. $\mathrm{Ca}$ dernos de Estudos Linguísticos 23, UNICAMP, Campinas, 1992. p: 18-55.

Recebido em: 10/04/2013; Aceito em: 15/06/2013 